
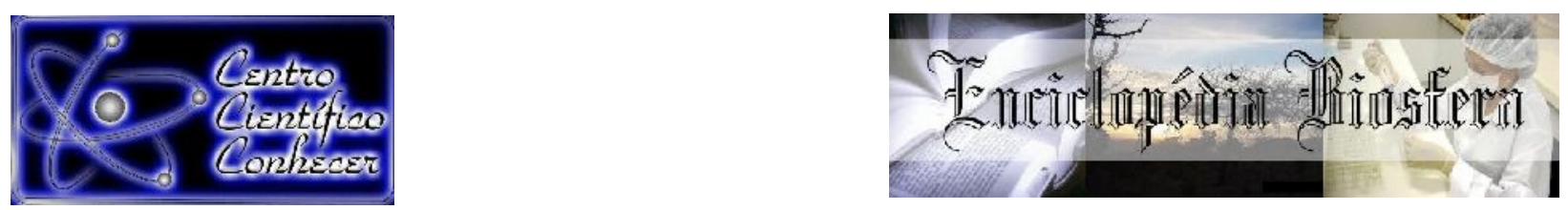

\title{
DESPERDÍCIO ZERO NA GESTÃO DE RESÍDUOS: CONCEITOS, DESAFIOS E TEMAS EMERGENTES A PARTIR DE UMA ANÁLISE DA PRODUÇÃO CIENTÍFICA
}

\author{
Rafaela da Silva Limons da Cunha ${ }^{1}$, Carlos Mello Garcias ${ }^{2}$ \\ ${ }^{1}$ Professora dos cursos de Engenharia Ambiental e Engenharia Civil; Doutoranda do \\ Programa de Pós-Graduação em Gestão Urbana da PUCPR, Curitiba-PR, Brasil \\ e-mail: rafaela.limons@pucpr.br \\ ${ }^{2}$ Professor Doutor do Programa de Pós-Graduação em Gestão Urbana da PUCPR, \\ Curitiba-PR, Brasil
}

Recebido em: 04/10/2019 - Aprovado em: 30/11/2019 - Publicado em: 15/12/2019 DOI: 10.18677/EnciBio 2019B21

Este estudo fornece uma revisão crítica da bibliografia sobre a temática Desperdício Zero, como forma de permitir uma análise do corpo dessa literatura com critérios de pesquisa rigorosa e reprodutíveis. O objetivo é destacar possíveis lacunas e identificar questões que possam representar áreas de investigação futura sobre o tema. A abordagem de pesquisa adotada foi a qualitativa, baseada na revisão sistemática, pois possibilitou aos pesquisadores a elaboração e o desenvolvimento do conhecimento em relação ao assunto Desperdício Zero de uma forma estruturada, clara e objetiva. A busca por artigos na base de dados Scopus possibilitou a identificação de um portfólio com 140 publicações a respeito do tema, e, após a aplicação dos critérios de triagem de artigos, foram selecionadas 38 publicações de revistas consideradas relevantes para esta pesquisa, para uma posterior etapa de apreciação. Os artigos foram avaliados utilizando dimensões estruturais específicas para agrupar a literatura nas seguintes categorias: Hierarquia na Gestão de Resíduos; Governança; e Indicadores de Sustentabilidade. Foram destacados como temas emergentes a necessidade de métodos para medição da prevenção de resíduos, a escassez de envolvimento de atores-chave na gestão e a dificuldade em se transformar a gestão linear de resíduos em uma gestão circular.

PALAVRAS-CHAVE: desperdício zero, governança, indicadores de sustentabilidade

\section{ZERO WASTE IN WASTE MANAGEMENT: CONCEPTS, CHALLENGES AND EMERGING THEMES FROM AN ANALYSIS OF SCIENTIFIC PRODUCTION}

\begin{abstract}
This study provides a critical review of the literature on the theme Zero Waste, as a way to allow an analysis of the body of this literature with rigorous and reproducible search criteria. The objective is to highlight possible gaps and identify issues that may represent future research areas on the topic. The research approach adopted
\end{abstract}


was the qualitative one, based on systematic review, as it allowed researchers to elaborate and develop knowledge regarding the Zero Waste subject in a structured, clear and objective manner. The search for articles in the Scopus database made it possible to identify a portfolio of 140 publications on the topic, and after applying the article screening criteria, 38 publications of journals considered relevant for this research were selected for further research. Appraisal stage. The articles were evaluated using specific structural dimensions to group the literature into the following categories: Hierarchy in Waste Management; Governance; and Sustainability Indicators. Emerging themes were highlighted the need for methods for measuring waste prevention, the lack of involvement of key actors in waste, and the difficulty of transforming linear waste management into circular management.

KEYWORDS: zero waste, governance, sustainability indicators

\section{INTRODUÇÃO}

As cidades são centros concentradores de produção e consumo, estando, assim, associadas a inúmeros impactos ambientais, sociais e econômicos, incluindo aqueles relacionados à produção de energia, as emissões de gases do efeito estufa, a geração de resíduos, ao aumento da poluição da água e a degradação ambiental local, regional e global (KOOP; VAN LEEUWEN, 2015).

Em relação a gestão de resíduos, muitas lacunas podem ser observadas no planejamento atual, visto que, estes sistemas não receberam tanta atenção no processo de planejamento da cidade quanto setores como de água ou energia (SONG et al., 2015).

O problema da geração, composição e destinação de resíduos municipais é de abrangência internacional, afetando, de forma distinta, tanto países desenvolvidos, em desenvolvimento e pobres. De forma geral, o crescimento populacional, a urbanização e o desenvolvimento econômico e tecnológico têm afetado os modos de produção, estilo de vida e consumo, resultando em aumento na quantidade e diversidade dos resíduos gerados (RODRIGUES et al., 2015)

A gestão de resíduos foi considerada no passado apenas como uma maneira de se livrar dos materiais residuais por aterro ou incineração, sendo que este ainda é o padrão dominante de descarte em todo o mundo, gerando uma enorme perda de recursos significativos e impactos ambientais elevados. Tem emergido uma nova possibilidade de se avaliar os resíduos, que reconhece a gestão como uma forma de recuperação de recursos e prevenção de impactos ambientais (GHISELLINI et al., 2016). Inclusive, entre os 17 Objetivos do Desenvolvimento Sustentável, que reflete os novos desafios dos Estados-membro das Nações Unidas, uma das metas é a redução substancial da geração de resíduos por meio da prevenção, redução, reciclagem e reuso até o ano de 2030.

A prevenção na geração está no topo da hierarquia do gerenciamento de resíduos e a necessidade de mudança de uma gestão linear (extração, produção, utilização e descarte) para uma gestão circular (aproveitamento dos resíduos como recursos para os diferentes processos) fez com que nos últimos anos, o conceito de desperdício zero tenha ganhado a atenção de instituições, estudiosos e empresas (WEETMAN, 2019).

Neste sentido, o objetivo geral deste estudo foi apresentar uma revisão crítica das publicações acadêmicas disponíveis que envolvam o conceito de Desperdício Zero. Utilizando o banco de dados Scopus com as palavras-chave definidas, e após aplicação dos filtros, foram selecionados 38 estudos para posterior análise. Esta 
revisão sistemática pode ser útil para gestores públicos que desenvolvam estratégias e políticas de desperdício zero, destacando tendências de pesquisa, e fornecendo diretrizes futuras de investigação potencial sobre o tema. Os artigos foram agrupados nas seguintes categorias: Hierarquia na Gestão de Resíduos; Governança e Indicadores de Sustentabilidade. Em cada categoria, grandes questões foram resumidas e insights importantes foram obtidos.

\section{MATERIAL E MÉTODOS}

A abordagem metodológica utilizada nesta pesquisa foi de revisão sistemática, cujo objetivo foi localizar e sintetizar a literatura sobre Desperdício Zero, por meio de procedimentos organizados, transparentes e replicáveis em cada etapa do processo, possibilitando uma compreensão abrangente do problema estudado (TRANFIELD et al., 2003; LITTELL et al., 2008; GALVÃO; PEREIRA, 2014). Este processo foi realizado entre janeiro e fevereiro de 2019 a partir da aplicação das palavras-chave da pesquisa dentro de uma base de dados de indexação de trabalhos.

O resumo das etapas do processo de revisão sistemática da literatura estão apresentados na Figura 1.

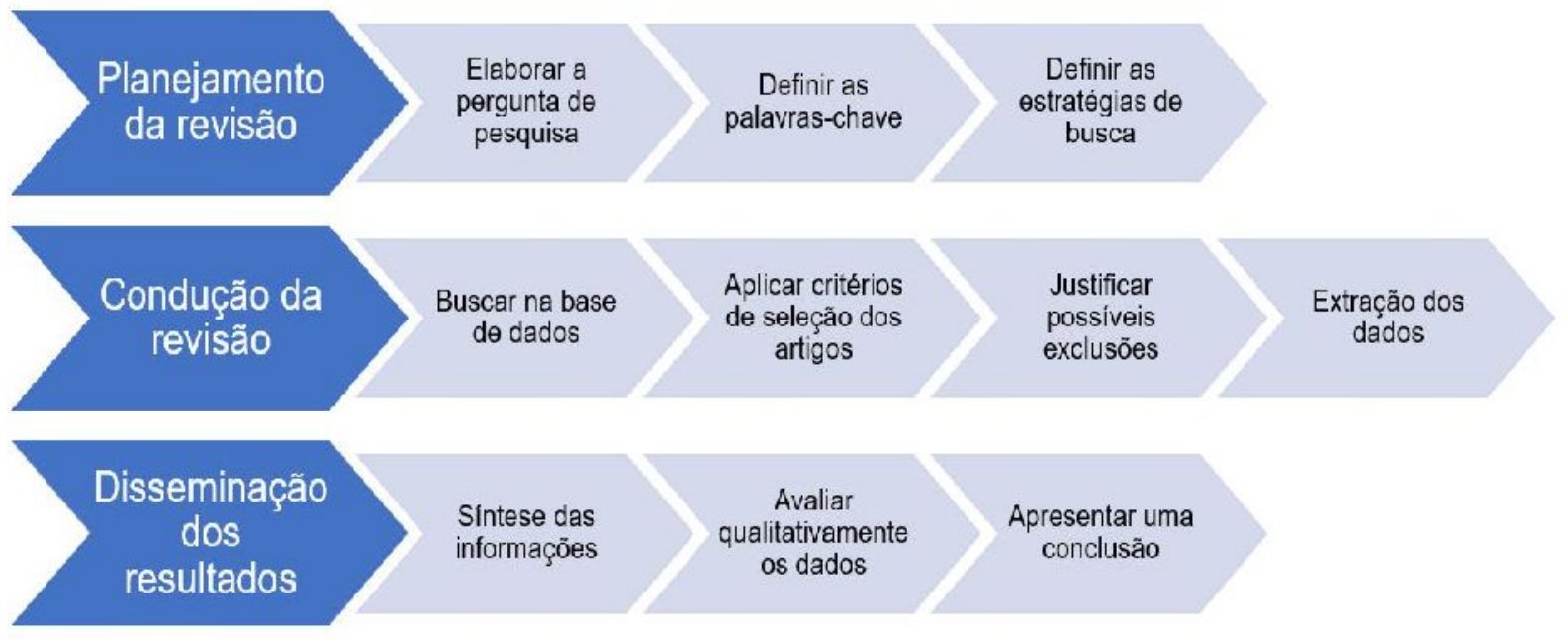

FIGURA 1 - Resumo das etapas do processo de revisão sistemática da literatura. Fonte: os autores (2019).

A primeira etapa da pesquisa se refere ao planejamento da revisão sistemática, que se inicia com a proposição de questões de pesquisa que possibilitem uma revisão ampla e identificação de estudos relevantes nas revistas científicas. As questões de pesquisa abordadas foram:

- Qual é o estado atual do conhecimento da temática Desperdício Zero na Gestão de Resíduos Municipais?

- Quais são as direções futuras da pesquisa?

Com base nestas questões foram definidas palavras-chave com o objetivo de coletar a literatura relevante sobre a problemática da pesquisa em diferentes contextos. Após uma busca prévia na literatura e de acordo com as questões de pesquisa as palavras-chave definidas foram: "zero waste" (desperdício zero, Desperdício Zero ou Lixo Zero), "municipal solid waste" (resíduo sólido municipal), 
"waste management" (gestão de resíduos) and "indicators" (indicadores) e o operador boleano "AND".

Foi definido como estratégia a busca na base de dados Scopus, e no que diz respeito à tipologia de resultado, indicou-se a modalidade "artigo". Não foi aplicado restrição cronológica e de idiomas, e após testes preliminares excluiu-se quatro áreas de pesquisa na base de dados: Chemistry; Medicine; Biochemistry, Genetics and Molecular Biology e Pharmacology, Toxicology and Pharmaceutics.

Diante do exposto, a segunda etapa da pesquisa foi a condução da revisão que se iniciou efetivamente com a busca na base de dados Scopus a partir da associação das palavras-chave duas a duas, três a três e por fim a associação de todas as palavras. Primeiramente foram identificados 226 artigos na soma das combinações das palavras-chave, que foram importados para o software Mendeley para eliminação de artigos duplicados, resultando, assim, em uma amostra de 140 artigos. Na sequência foram selecionados os artigos conforme os critérios de inclusão e exclusão estabelecidos:

a) Critérios de inclusão: foram incluídos todos os artigos que discutiam a temática central correlacionada com Desperdício Zero, mesmo que não estivessem relacionados diretamente à gestão de resíduos municipais, mas que apresentassem conceitos, recomendações e/ou limitação da sua aplicação, que poderiam servir como base para pesquisas futuras;

b) Critérios de exclusão: foram excluídos os artigos que tratavam dos processos físicos, químicos e biológicos dos tratamentos de resíduos; publicações cujo conteúdo não estava disponível para leitura e aqueles artigos que não tratavam da temática Desperdício Zero ou que apresentavam uma discussão muito superficial sobre o tema.

Após a aplicação desses critérios, foram reunidas 35 publicações de revistas consideradas relevantes para esta pesquisa para posterior etapa de avaliação. Além disso, esses artigos foram lidos para verificar no corpo da literatura selecionada as referências mais relevantes, resultando em outros três artigos, complementando a amostra inicial do Scopus.

O processo de coleta resultou em um total de 38 documentos, que foram utilizados para extração de dados. A Figura 2 apresenta um resumo destas duas primeiras etapas que foram seguidas para seleção dos artigos desta revisão sistemática. 


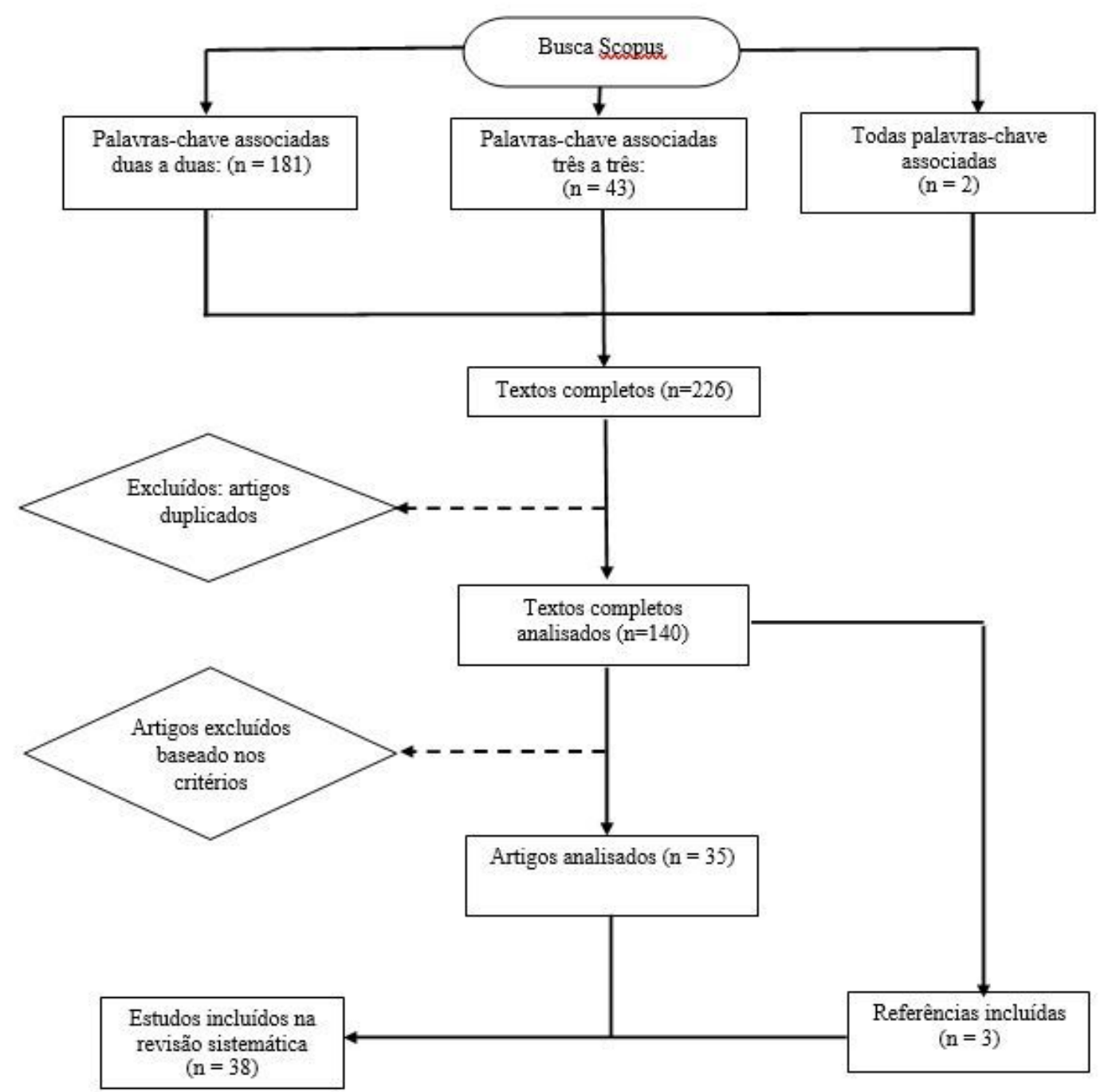

FIGURA 2 - Processo de definição de artigos. Fonte: os autores (2019).

Finalmente, a terceira etapa da pesquisa se refere a disseminação dos resultados, que é a etapa mais importante da revisão, gerando conhecimento baseado na coleta e na análise dos dados. Foi realizada a leitura dos textos na íntegra com uma apreciação qualitativa das informações. Os resultados e a discussão são apresentados de forma descritiva, por meio da exposição dos dados relativos às publicações e da análise de conteúdo desses materiais. Para melhor organização das informações, os resultados encontrados nos artigos selecionados foram classificados em três categorias, de acordo com quais tópicos eles discutiam em profundidade. Conforme observado na Figura 3, as categorias analisadas foram: Categoria 1: Hierarquia na Gestão de Resíduos; Categoria 2: Governança na Gestão de Resíduos e Categoria 3: Indicadores de Sustentabilidade. 


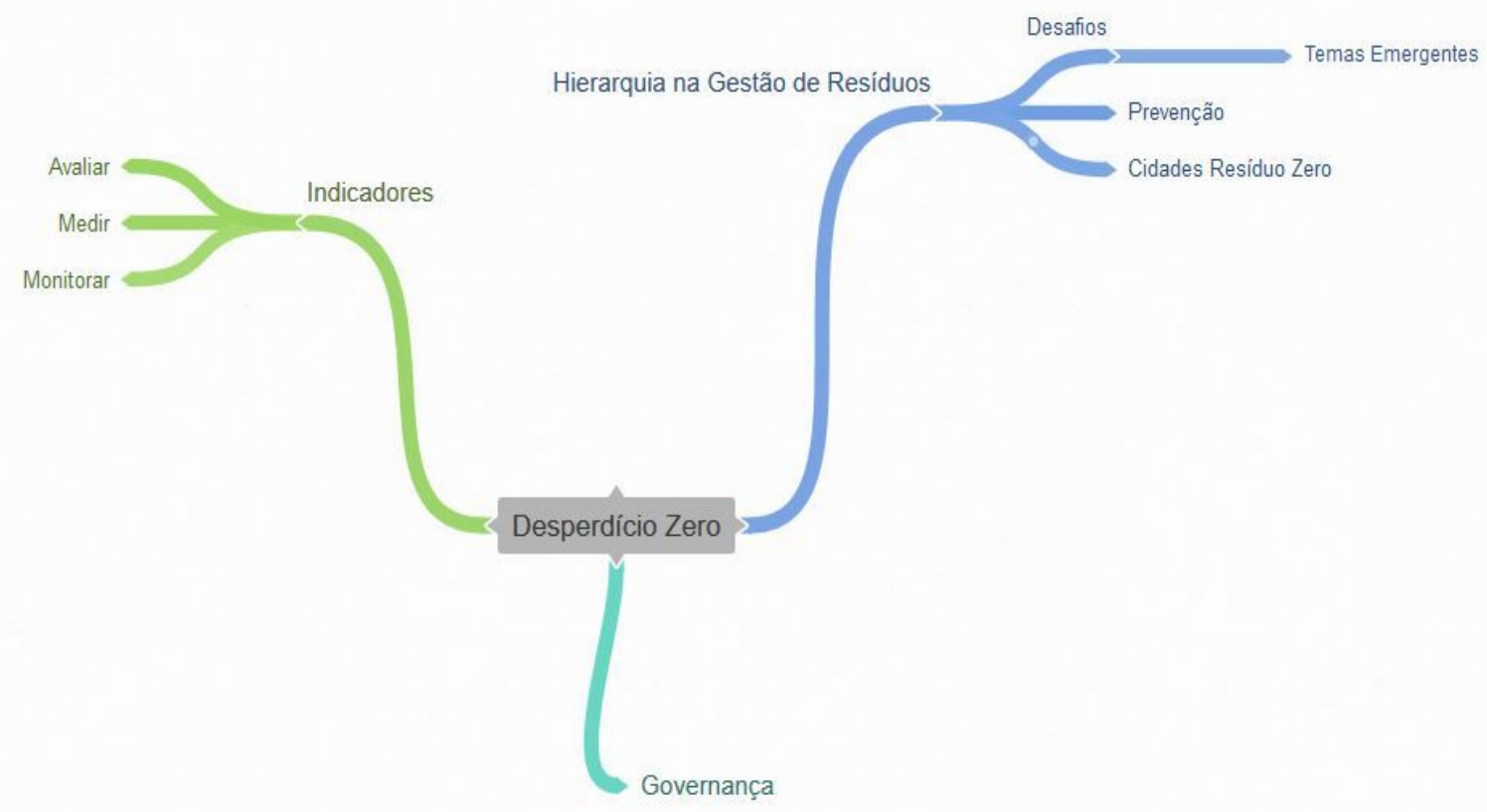

FIGURA 3 - Mapa mental com as categorias analisadas. Fonte: os autores (2019).

\section{RESULTADOS E DISCUSSÃO}

Foram analisados 35 artigos publicados entre 1975 e 2018, e nesta etapa são apresentados os resultados da avaliação qualitativa dos artigos, a partir de uma síntese de informação dos resultados que foram considerados mais relevantes. As características dos estudos analisados serão apresentadas e discutidas de acordo com categorias definidas da metodologia desta pesquisas. Essas categorias foram escolhidas pois apontaram questões de destaque na literatura revisada. Por terem temas abrangentes, destacaram questões relacionadas à conceituação, apresentando relação com o objetivo da pesquisa.

Com base nesta análise qualitativa, a Figura 4 apresenta uma nuvem de palavras com os termos com maior ocorrência nos artigos analisados, que serão apresentados de forma mais detalhado na sequência.

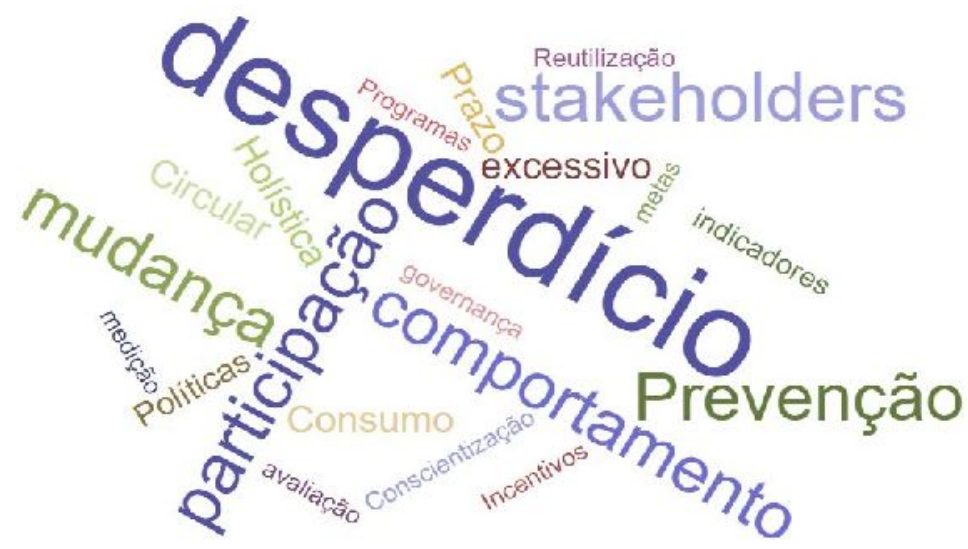

FIGURA 4 - Nuvem de palavras com os termos com maior ocorrência. Fonte: os autores (2019). 


\section{Categoria 1: Hierarquia na Gestão de Resíduos}

Nesta primeira categoria serão apresentados aspectos em relação ao Desperdício Zero discutido por diferentes pesquisadores, salientando a necessidade de adoção de uma abordagem preventiva, holística e circular dos materiais, ou seja, indicando que o caminho a ser seguido não pode ser com foco em como se tratar os resíduos produzidos, mas sim em como não os produzir. Neste contexto, o gerenciamento de resíduos é de fato desenvolvido seguindo a hierarquia de gerenciamento de resíduos e, portanto, deve depender não apenas da eliminação de resíduos, mas primordialmente da prevenção e reutilização.

A hierarquia de gerenciamento de resíduos é um guia nacional e internacionalmente aceito para priorizar práticas de gestão de resíduos com o objetivo de alcançar resultados ambientais e utilização de recursos ideais (SONG et al., 2015). A Diretiva 2008/98/CE da União Europeia, no Artigo $4^{\circ}$, estabelece como Hierarquia a ser considerada na gestão dos resíduos os aspectos apresentados na Figura 5.

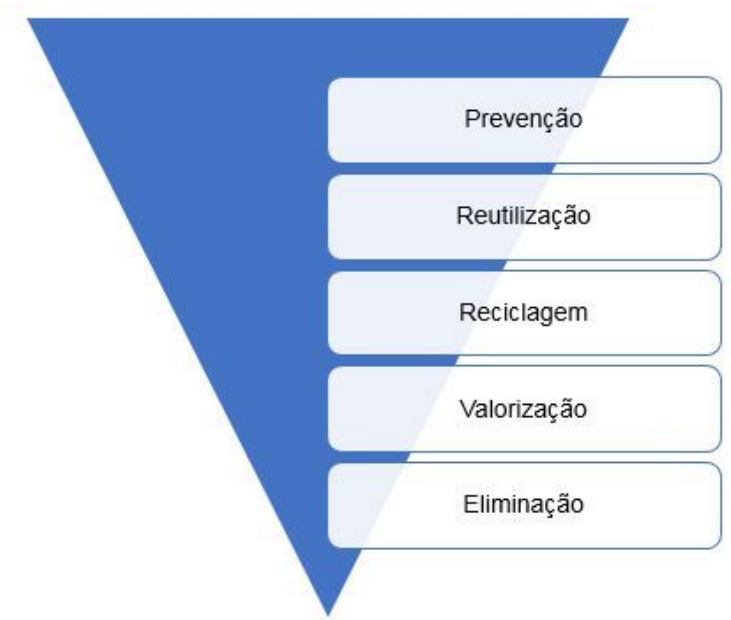

FIGURA 5 - Hierarquia no Gerenciamento de Resíduos. Fonte: Adaptado de Diretiva 2008/98/CE.

Segundo a Diretiva 2008/98/CE, a prevenção de resíduos é definida como "as medidas tomadas antes de uma substância, material ou produto tornar-se resíduo, reduzir a quantidade de resíduos, os impactos adversos gerados na saúde ambiental e humana ou o conteúdo de substâncias nocivas". Conforme indicado por Zorpas e Lasiridi (2013) a prevenção de resíduos inclui uma prevenção rigorosa na geração; na redução qualitativa e quantitativa na fonte; e na reutilização do produto., ou seja, aquilo que não pudesse ser prevenido, seguiria a sequência da hierarquia, investindo em métodos de reciclagem; em métodos de valorização dos resíduos, como, por exemplo, a valorização energética; e, por último, somente os rejeitos que não apresentam nenhuma outra possibilidade, seriam encaminhados para a eliminação, como, por exemplo, aterros sanitários.

O Desperdício Zero é uma abordagem de todo o sistema que visa eliminar, em vez de gerenciar os resíduos, ou seja, ele rejeita o uso atual de recursos unidirecionais de uso linear e a cultura de descarte, sendo a favor de um sistema circular de "circuito fechado". De acordo com Boyle (2000), o Desperdício Zero pode significar um caminho econômico aos sistemas de resíduos, em que novos recursos 
são continuamente necessários para reabastecer as matérias-primas desperdiçadas e uma alternativa ambiental, uma vez que os resíduos constituem uma quantidade significativa de poluição no mundo.

Segundo Zaman (2015) a necessidade de mudança da gestão linear de resíduos fez com que o conceito de Desperdício Zero emergisse na última década como um método inovador, que reconhece que os recursos que são transformados em resíduos como resultado das atividades humanas devem ser redirecionados para o processo de produção a partir de sistemas circulares de gerenciamento.

Muitas vezes esse conceito tem sido implementado nas sociedades como uma meta de zero resíduos enviados para aterro e incineração, ou uma meta de aumento nas taxas de reciclagem, sem se avaliar a necessidade de soluções de políticas de longo prazo para evitar o consumo excessivo, sendo assim, considerado um mecanismo reducionista, pois não permite uma solução holística de longo prazo, com questionamentos acerca das questões de redução e reutilização na fonte (ZAMAN; LEHMAN, 2013; ZAMAN, 2014; ZAMAN, 2015; SONG et al., 2015; ILLIC; NIKOLIC, 2016; ZAMAN, 2016; ZAMAN; SWAPAN, 2016; SILVA et al., 2016).

É preciso uma reformulação dos paradigmas para alterar a base de soluções de tratamento e eliminação de resíduos (LEHMAN, 2011; LEHMAN et al., 2013a; LEHMAN et al., 2013b). Assim, para se tratar efetivamente do problema do desperdício e avançar em direção a um sistema holístico, "deve haver um movimento além da reciclagem para o território amplamente desconhecido da extremidade superior da hierarquia de gerenciamento de resíduos, para reutilização, redução e prevenção" (SONG et al., 2015).

Com base na avaliação dos artigos observou-se que os principais desafios identificados para se conseguir essa transformação de uma gestão linear de resíduos para práticas de Desperdício Zero são: necessidade de mudança de comportamento e a participação ativa dos stakeholders na gestão de resíduos; necessidade de legislações específicas e desenvolvimento de medidas e metas para monitoramento das ações de prevenção de resíduos.

O desafio de participação ativa dos agentes interessados na gestão de resíduos foi identificado nos principais artigos analisados (BOYLE, 2000; ZAMAN; LEHMAN, 2011; VELEVA et al., 2017, INDROSAPTONO; SYAHBANA, 2017; VELENTURF et al., 2018; LYNCH et al., 2018; MARTINHO et al., 2018) e Zaman (2014) um dos autores mais relevantes nesta temática corrobora este aspecto considerando que os benefícios ambientais e econômicos dos sistemas de gestão de resíduos poderiam ser alcançados a partir do aprimoramento e consequente envolvimento da comunidade dos programas locais de gestão de resíduos.

A necessidade de legislações especificas de esgotamento zero e políticas de incentivo e regulamentos adequados foram identificados em diversos estudos analisados (BOYLE, 2000; ZAMAN; LEHMAN, 2013; ILIC; NIKOLIC; 2016; VELENTURF et al., 2018). Estes estudos indicam a necessidade de regras e incentivos que priorizem o conceito de resíduo como um recurso a ser valorizado, e não somente como um material nocivo a ser descartado.

Veleva et al. (2017) consideram a necessidade do desenvolvimento de indicadores eficazes que são essenciais para aumentar a conscientização e capacitar os agentes envolvidos na gestão do desperdício zero. O desafio da necessidade de desenvolver melhores medidas e metas para avançar em direção ao Desperdício Zero a fim de que se possa monitorar os esforços de redução de resíduos foram observados em diferentes artigos que consideram este como uma 
das lacunas de pesquisa sobre a temática em questão (ZORPAS; LASARIDI, 2013; ZAMAN; LEHMAN, 2013; ZAMAN, 2014; ZAMAN, 2016; ZAMAN; SWAPAN, 2016; VELEVA et al., 2017; SAHIMAA et al., 2017; ; MARTINHO et al., 2018; VELENTURF et al., 2018).

Há consenso entre os estudos analisados que estabelecer metas para desviar os resíduos dos aterros sanitários e aumentar as taxas de reciclagem e recuperação, atendendo o que preconiza a hierarquia de gerenciamento de resíduos é um grande desafio para a sociedade, governo e setores produtivos.

\section{Categoria 2: Governança na Gestão de Resíduos}

A análise dos estudos selecionados indicou a governança na gestão de resíduos sólidos como um dos desafios da implementação do Desperdício Zero, e foram observados poucos estudos que analisaram este aspecto de forma aprofundada.

Dentre as pesquisas identificadas, Silva et al. (2016) foram um dos primeiros pesquisadores que analisaram o aspecto da governança para a gestão de resíduos com foco no Desperdício Zero. Segundo os pesquisadores este conceito é implementado na maioria das vezes a partir de modelos de governança em "três níveis", que foram construídos para gerenciar fluxos de materiais lineares. No entanto, para se abordar de forma holística os desafios da prevenção de resíduos, o modelo de governança atual precisará ser expandido no intuito de incluir uma multiplicidade de atores adicionais.

Wong (2017) utilizou-se de uma abordagem qualitativa e de entrevistas para discutir e analisar a governança ambiental em nível local no distrito de Beitou (Taiwan), enfocando o descontentamento social dos cidadãos sobre a construção de um incinerador em sua comunidade e a necessidade da promoção de uma cidade de desperdício zero. A autora indica que a interação entre atores estatais e não estatais tem demonstrado a abordagem da governança participativa a nível local em Taiwan e tem sido uma solução para os desafios ambientais locais. No entanto, considera ainda como limitações o fato de os cidadãos ainda não terem um papel no processo de elaboração de políticas ambientais, além da falta de transparência e da exclusão dos cidadãos do processo decisório.

A necessidade de participação de atores-chave na gestão de resíduos foi identificada por Aguiñagua et al. (2018) que propõem uma estrutura de governança de baixo para cima, a fim de expandir a gestão circular. Os autores apresentam como desafios dessa abordagem a necessidade de que os atores sociais precisem compartilhar suas ideias e "obstáculos existindo confiança, reciprocidade e colaboração entre os envolvidos, e também a necessidade de incentivar empreendedores sustentáveis a aproveitar as oportunidades de criação de bens e serviços consistentes com o desenvolvimento sustentável.

Velenturf et al. (2018), a partir de uma pesquisa realizada no Reino Unido, consideraram que a participação de parceiros governamentais em múltiplos níveis foi crucial no processo de transição para uma gestão mais sustentável de resíduos, e que o contato regular com as organizações governamentais permite 0 fornecimento das recomendações oportunas e concretas necessárias no processo de governança. Os autores também ponderam a importância da parceria entre universidades e organizações governamentais para o sucesso dessa transição.

Lynch et al. (2018) realizaram uma discussão com grupos focais em 27 países da União Europeia, atentando-se às barreiras e às preocupações com 
relação à gestão de resíduos. Como resultados, esses grupos propuseram e priorizaram mais de 350 ideias para se chegar em uma sociedade com "desperdício zero", e os autores argumentam que o envolvimento dos cidadãos na agenda de inovação é viável e conduz a contribuições significativas.

$\mathrm{Na}$ análise destas pesquisas foram identificadas possibilidades de inovação entre os stakeholders da cadeia de resíduos, considerando que o foco deve ser dado na governança, ou seja, deve-se transferir para todos os envolvidos a responsabilidade de se resolver o problema. Assim, é imprescindível que seja aplicado um modelo de governança que alinhe a estratégia de desperdício zero com as expectativas dos atores envolvidos.

\section{Categoria 3: Indicadores de Sustentabilidade}

Esta última categoria inclui publicações que tratam da necessidade de indicadores de sistemas de desperdício zero, que serão fundamentais para que políticos e tomadores de decisão possam medir, monitorar e avaliar esses sistemas complexos, tendo em vista o desenvolvimento futuro. A Diretiva 2008/98/CE estabeleceu procedimentos claros de prevenção de resíduos, incluindo a apresentação de relatórios, a revisão, o acompanhamento e a avaliação.

Veleva et al. (2017) consideram que as empresas apresentam um papel fundamental no movimento em direção ao "desperdício zero". No entanto, sugerem que os esforços atualmente são inadequados, pois não possuem indicadores de sustentabilidade efetivos para medir o progresso, para identificar oportunidades e para envolver os funcionários. Os autores propõem um modelo para a prática de "desperdício zero", apresentando como aspecto inovador do modelo a incorporação do engajamento dos funcionários como elemento-chave da estratégia. Os autores concluem e apresentam como principal contribuição da pesquisa a necessidade do desenvolvimento de indicadores eficazes para o avanço das práticas circulares econômicas, e mesmo considerando que os indicadores por si só não possam trazer mudanças, eles são enfáticos quanto à imprescindibilidade de se elevar a conscientização e a capacitação dos atores sociais envolvidos no avanço para uma economia com Desperdício Zero.

Zorpas e Lasaridi (2013) discutem que medir a prevenção de resíduos é um processo complexo e difícil. Porém, como tem se tornado cada vez mais importante para o gerenciamento de resíduos, tanto no nível de planejamento como no de implementação, é fundamental que se desenvolvam métodos confiáveis para monitorar, medir e avaliar a prevenção de resíduos e seus benefícios, bem como avaliar a eficácia das ações, com o objetivo de promover uma conscientização relevante que gere mudanças comportamentais.

De acordo com Zaman (2014), a precisão da ferramenta de avaliação de desempenho é muito dependente de um conjunto de indicadores ou variáveis confiáveis que refletem a realidade. Ainda de acordo com Zaman (2014), é importante identificar os indicadores-chave para desenvolver ferramentas e estratégias de Desperdício Zero no futuro, visto que, de acordo com Zorpas e Lasaridi (2013), o monitoramento feito corretamente é uma ferramenta inestimável para uma boa gestão e fornece uma base útil para avaliação

Os desafios de previsão de resíduos incluem alcançar indicadores de desperdício de mudança em sistemas dinâmicos, estabelecer metas de sustentabilidade para as quais o progresso de resíduos possa ser medido, e fornecer incentivos positivos para novos esforços. Esses indicadores são muito úteis 
para analisar o progresso e para relatar o status dos resíduos à autoridade local (ZAMAN, 2014).

Zaman e Lehman (2013) desenvolveram uma ferramenta chamada Zero Waste Index (ZWI) para medir os recursos naturais recuperados de resíduos sólidos urbanos e, além disso, estimar a economia de energia, gás de efeito estufa e também de água, compensando materiais virgens e recuperando energia de resíduos Os autores testaram a ferramenta em duas cidades, Adelaide e Estocolmo, e concluíram que essa ferramenta ajuda os planejadores a pensar em um uso mais eficiente de materiais, permitindo maior reciclagem. Sendo assim, é considerado como um indicador de desempenho para avaliar o desempenho global dos sistemas de gerenciamento de resíduos.

Em uma nova pesquisa, Zaman (2016) mediu o desempenho atual dos sistemas de gerenciamento de resíduos urbanos em 172 países, aplicando a ferramenta citada anteriormente: ZWI. O autor concluiu que a recuperação global dos recursos e o desempenho da gestão de resíduos são significativamente baixos, e que os benefícios ambientais e econômicos globais dos sistemas de gestão de resíduos poderiam ser alcançados a partir do aprimoramento e envolvimento dos atores sociais nos programas locais de gestão de resíduos.

Zaman e Swapan (2016) também aplicaram a ferramenta ZWI com o objetivo de apresentar os benefícios ambientais e econômicos dos sistemas globais de gerenciamento de resíduos, aplicando as práticas de Desperdício Zero em 168 países. Os resultados indicaram que, globalmente, a pessoa média gerou cerca de $435 \mathrm{~kg}$ de resíduos por ano. Também estimaram que $50 \mathrm{~kg}$ de materiais (papel, plástico, metal, vidro e outros) potencialmente substituem a demanda pela extração de materiais virgens. Ao substituir a demanda por materiais virgens, através das atividades de Desperdício Zero, a pessoa média poderia economizar cerca de $216 \mathrm{kWh}$ de energia, $0,05 \mathrm{~kg}$ de GEE e $36 \mathrm{~L}$ de água processada. O estudo também concluiu que dados confiáveis e precisos sobre resíduos são um dos principais desafios na classificação do desempenho global de gerenciamento de resíduos, já que vários países usam técnicas assimétricas de medição e de relatório.

Zaman (2014) identifica os principais indicadores de Desperdício Zero que poderiam ser utilizados para avaliar o desempenho dos sistemas de gerenciamento de resíduos zero. Com base em uma extensiva revisão da literatura, o autor categoriza os indicadores de Desperdício Zero em sete categorias: geoadministrativa, sociocultural, administrativa, econômica, ambiental, organizacional e política. Os 238 indicadores selecionados pelo autor foram encaminhados para especialistas da área, que os classificaram de "não necessário" para "muito alta prioridade", finalizando com 56 indicadores que foram considerados como os mais importantes para os sistemas de gestão de resíduos. O autor justifica a importância do trabalho indicando que sempre foi um desafio para os tomadores de decisão avaliar efetivamente o progresso das estratégias e ferramentas de gerenciamento de resíduos, e que, apesar de existirem vários estudos disponíveis sobre indicadores de resíduos e ferramentas de avaliação, os indicadores de avaliação de resíduos zero ainda não foram identificados de forma holística, concluindo que é necessário o desenvolvimento de ferramentas de avaliação de Desperdício Zero com base nos indicadores propostos. 


\section{CONCLUSÃO}

A análise da produção científica sobre o tema proposto forneceu uma visão abrangente sobre os principais conceitos e princípios do Desperdício Zero, sendo possível concluir que é um tema atual e visionário, embora ainda existam algumas confusões em relação ao seu fundamento. Um dos principais desafios identificados nesta revisão é o de estabelecer o Desperdício Zero como uma abordagem preventiva, holística e circular dos materiais, e não somente como meta zero de resíduos enviados para aterro e incineração.

Há consenso entre os estudos analisados sobre a necessidade de mudança de comportamento dos agentes envolvidos na gestão de resíduos e da importância de legislações específicas, incentivos e programas visando a compreensão do custo econômico, ambiental e social quando não se considera de forma adequada a hierarquia de gerenciamento de resíduos com foco primordial na prevenção da geração de resíduos.

Este estudo identificou como temas emergentes e possíveis gaps de pesquisa, a importância do envolvimento de stakeholders na gestão de resíduos com foco no desperdício zero, além da necessidade do desenvolvimento de indicadores para avaliação do desperdício e prevenção de resíduos.

Por fim, espera-se que os resultados apresentados neste trabalho permitam uma melhor compreensão das principais barreiras, desafios na busca de uma abordagem preventiva e circular na gestão de resíduos sólidos. No entanto, não foram considerados todos os estudos relacionados ao tema, visto que a definição das palavras-chave, critérios de inclusão e exclusão, constituem umas das possíveis formas de se analisar a temática em questão. Uma sugestão para trabalhos futuros seria uma nova revisão sistemática combinando a palavra-chave "Desperdício Zero" com os termos de maior ocorrência identificados na nuvem de palavras desta pesquisa.

\section{REFERÊNCIAS}

AGUIÑAGA, E.; HENRIQUES, I.; SCHEEL, C.; SCHEEL, A. Building resilience: A self-sustainable community approach to the triple bottom line. Journal of Cleaner Producton, v.173, n.1, p.1860196, $2018 . \quad$ Disponível: <https://doi.org/10.1016/j.jclepro.2017.01.094>. doi: 10.1016/j.jclepro.2017.01.094

BOYLE, C.A. Solid waste management in New Zealand. Waste Management, v.20, n.7, p. 517-526, 2000. Disponível em: <https://doi.org/10.1016/S0956053X(00)00023-4>. doi: 10.1016/S0956-053X(00)00023-4

\section{DIRECTIVA 2008/98/CE DO PARLAMENTO EUROPEU E DO CONSELHO.} Disponível em: <http://data.europa.eu/eli/dir/2008/98/oj>.

GHISELLINI, P.; CIALANI, C.; UGLIATI, S. A review on circular economy: the expected transition to a balanced interplay of environmental and economic systems. Journal of Cleaner Production, v. 114, p.11-32, 2016. Disponível em: <https://doi.org/10.1016/j.jclepro.2015.09.007>. doi: 10.1016/j.jclepro.2015.09.007

ILLIC, M.; NIKOLIC, M. Drivers for development of circular economy - A case study of Serbia. Habitat International, v.56, p.191-200, 2016. Disponível em: 
<https://doi.org/10.1016/j.habitatint.2016.06.003>.

doi:

0.1016/j.habitatint.2016.06.003

INDROSAPTONO, D.; SYAHBANA, A. Informal sector in urban inorganic waste management toward $3 \mathrm{M}$ management (Merubah: Changing, Mengurangi: Reducing, Manfaat: Benefit) in Semarang city. Journal of Architecture and Urbanism, v. 41, p. 278-287, 2017. Disponível em:< https://doi.org/10.3846/20297955.2017.1411849>.

doi: 10.3846/20297955.2017.1411849

GALVAO, T. F.; PEREIRA, M. G. Revisões sistemáticas da literatura: passos para sua elaboração. Epidemiologia e Serviços de Saúde, v. 23, n. 1, p. 183-184, 2014. Disponível em:< https://dx.doi.org/10.5123/S1679-49742014000100018>. doi: 10.5123/S1679-49742014000100018

KOOP, S.H.A.; VAN LEEUWEN, C.J. Application of the improved city blueprint framework in 45 municipalities and regions. Water Resources Manegement, v. 29, p. 4629-4647, 2015. Disponível em:<https://doi.org/10.1007/s11269-015-1079-7>. doi: 10.1007/s11269-015-1079-7

LEHMAN, S. Optimzing urban material flows and waste streams ins urban development through principles of zero waste and sustainable consumption. Sustainability, v.3, p. 155-183, 2011. Disponível em:<https://doi.org/ 10.3390/su3010155>. doi: 10.3390/su3010155

LEHMANN, S.; ZAMAN, A.U.; DEVLIN, J. Integrated demand forecasting to support urban planning of low carbon precints: the waste scenario. Industry Corner, v.8, n.2, p. 54-70, 2013a. Disponível em:< https://doi.org/10.3992/jgb.8.2.54>. doi: 10.3992/jgb.8.2.54

LEHMAN, S.; ZAMAN, A.U.; DEVLIN, J.; HOLYOAK, N. Supporting urban planning of low-carbon precints: integrated demand forescasting. Sustainability, v.5, p.52895318, 2013b. Disponível em:<https://doi.org/10.3390/su5125289>. doi: $10.3390 /$ su5125289

LITTEL, J.H.; CORCORAN, J.; PILLAI, V.K. (2008). Systematic Reviews and MetaAnalysis. Oxford University Press, USA.

LYNCH, D.; KUPPER, F.; BROERSE, J. Toward a socially desirable EU research and innovation agenda on urban waste: A transnational EU citizen consultation. Sustainability, v.10, n.10, p.1641, 2018. Disponível em:<https://doi.org /10.3390/su10051641>. doi: 10.3390/su10051641

MARTINHO, G.; GOMES, A.; RAMOS, M.; SANTOS, P.; GONÇALVES, G. et al. Solid waste prevention and management at green festivals: A case study of the Andanças Festival, Portugal. Waste Management, v.71, p.10-18, 2018. Disponível em: $\quad$ <https://doi.org/10.1016/j.wasman.2017.10.020>. 10.1016/j.wasman.2017.10.020

RODRIGUES, W.; MAGALHÃES FILHO, L.N.L.; PEREIRA, R.S. Análise dos determinantes dos custos de resíduos sólidos urbanos nas capitais estaduais 
brasileiras. Revista Brasileira de Gestão Urbana, v.8, n.1, p.130-141, 2015. Disponível em: <http://dx.doi.org/10.1590/2175-3369.008.001.AO02>. doi: 10.1590/2175-3369.008.001.AO02.

SAHIMAA, O.; MATTINEN, M.K.; KOSKELA, S.; SALO, M.; SORVARI, J. et al. Towards zero climate emissions, zero waste, and one planet living - Testing the applicability of three indicators in Finnish cities. Sustainable Production and Consumption, v.10, p.121-132, 2017. Disponível em:< https://doi.org/10.1016/j.spc.2017.02.004>. doi: 10.1016/j.spc.2017.02.004

SILVA, A.; STOCKER, L.; MERCIECA, P.; ROSANA, M.; The role of policy labels, keywords and framing in transitioning waste policy. Journal of Cleaner Production, v.115, n. 1, p.224-237, 2016. Disponível em: $<$ https://doi.org/10.1016/j.jclepro.2015.12.069>. 10.1016/j.jclepro.2015.12.069silva

SONG, Q.; LI, J.; ZENG, X. Minimizing the increasing solid waste through zero waste strategy. Journal of Cleaner Production, v. 104, n.1, p.199-210, 2015. Disponível em: $\quad$ <https://doi.org/10.1016/j.jclepro.2014.08.027>. doi: 10.1016/j.jclepro.2014.08.027

TRANFIELD, D.; DENYER, D.; SMART, P. Towards a methodology for developing evidence-informed management knowledge by means of systematic review. British of Management, v.14, p.207-22, 2003. Disponível em:<https://doi.org/10.1111/14678551.00375>. doi: https://doi.org/10.1111/1467-8551.00375

VELEVA, V.; BODKIN, G.; TODOROVA, S. The need for better measurement and employee engagement to advance a circular economy: Lessons from Biogen's "Zero Waste" journey". Journal of Cleaner Production, v.154, p. 517- 529, 2017. Disponível em: <https://doi.org/10.1016/j.jclepro.2017.03.177>. doi: 10.1016/j.jclepro.2017.03.177

VELENTURF, A.; PURNELL, P.; TREGENT, M.; FERGUSON, J.; HOLMES, A. Producing a Vision and Approach for the Transition towards a Circular Economy: Perspectives from Government Partners. Sustainability, v.10, n.5, p. 1401, 2018. Disponível em:<https://doi.org/10.3390/su10051401>. doi: 10.3390/su10051401

WEETMAN, C. Economia circular: conceitos e estratégias para fazer negócios de forma mais inteligente, sustentável e lucrativa. Tradução de Afonso Celso da Cunha Serra. 1. ed. São Paulo: Autêntica Business, 2019. 512 p.

WONG, N. W.M. The road to environmental participatory governance in Taiwan: collaboration and challenges in incineration and municipal waste management. Journal of Environmental Planning and Management, v.60, n.10, p.1726-1740, 2017. Disponível em:<https://doi.org/10.1080/09640568.2016.1251400>. doi: 10.1080/09640568.2016.1251400

ZAMAN, A. U. Measuring waste management performance using the 'Zero Waste Index': the case of Adelaide, Australia. Journal of Cleaner Production, v.66, p.407- 
419, 2014. Disponível em: <https://doi.org/10.1016/j.jclepro.2013.10.032>. doi: 10.1016/j.jclepro.2013.10.032

ZAMAN, A. U. A comprehensive review of the development of zero waste management: lessons learned and guidelines. Journal of Cleaner Production, v. 91, p. 12-25, 2015. Disponível em: <https://doi.org/10.1016/j.jclepro.2014.12.013>. doi: $10.1016 /$ j.jclepro.2014.12.013

ZAMAN, A.U. A comprehensive study of the environmental and economic benefits of resource recovery from global waste management systems, Journal of Cleaner Production, v.124, p.41-50, $2016 . \quad$ Disponível em:<https://doi.org/10.1016/j.jclepro.2016.02.086> doi: 10.1016/j.jclepro.2016.02.086

ZAMAN, A. U.; LEHMANN, S. Urban growth and waste management optimization towards "zero waste city." City Culture and Society, v. 2, n. 4, p. 177-187, 2011. Disponível em: <https://doi.org/10.1016/j.ccs.2011.11.007>. doi: 10.1016/j.ccs.2011.11.007

ZAMAN, A. U.; LEHMANN, S. The zero waste Index: A performance measurement tool for waste management systems in a "Zero waste city", v. 50, p. 123-132, 2013. Disponível em:< https://doi.org/10.1016/j.jclepro.2012.11.041>. doi: 10.1016/j.jclepro.2012.11.041

ZAMAN, A.U.; SWAPAN, M.S.H. Performance evaluation and benchmarking of global waste management systems. Resources, Conservation and Recycling, v. 114, p.32-41, 2016. Disponível em: <https://doi.org/10.1016/j.resconrec.2016.06.020>. doi: 10.1016/j.resconrec.2016.06.020

ZORPAS, A.A.; LASARIDI, K. Measuring waste prevention. Waste Management, v. 33, n. 5, p.1047-1056, 2013. Disponível em: <https://doi.org/10.1016/j.wasman.2012.12.017>. doi: 10.1016/j.wasman.2012.12.017 